\title{
BMJ Open Association between dietary antioxidant vitamins intake and homocysteine levels in middle-aged and older adults with hypertension: a cross-sectional study
}

Xiaolin Peng, ${ }^{1}$ Qin Gao, ${ }^{2,3}$ Juan Zhou, ${ }^{2}$ Jianping Ma, ${ }^{1}$ Dan Zhao, ${ }^{1}$ Liping Hao (D ${ }^{2}$

To cite: Peng X, Gao Q, Zhou J, et al. Association between dietary antioxidant vitamins intake and homocysteine levels in middle-aged and older adults with hypertension: a crosssectional

study. BMJ Open

2021;11:e045732. doi:10.1136/ bmjopen-2020-045732

- Prepublication history and additional supplemental material for this paper are available online. To view these files, please visit the journal online (http://dx.doi.org/10.1136/ bmjopen-2020-045732).

XP and QG contributed equally.

Received 12 October 2020 Accepted 24 September 2021

Check for updates

(C) Author(s) (or their employer(s)) 2021. Re-use permitted under CC BY-NC. No commercial re-use. See rights and permissions. Published by BMJ.

For numbered affiliations see end of article.

Correspondence to

Dr Liping HaO;

haolp@mails.tjmu.edu.cn

\section{ABSTRACT}

Objectives Plasma total homocysteine (tHcy) has been implicated in the development of cardiovascular disease. This study aimed to assess the relationship of dietary antioxidant vitamins intake with tHcy levels in middle-aged and older adults with hypertension.

Design A cross-sectional study.

Setting The survey was conducted in the Nanshan district of Shenzhen.

Participants A total of 1465 middle-aged and older adults with hypertension were included between July and September of 2013.

Measurements Hyperhomocysteinaemia (HHcy) was defined as tHcy $\geq 15 \mu \mathrm{mol} / \mathrm{L}$. Some dietary antioxidant vitamins (vitamin C (VC) and vitamin E (VE), carotenes, retinol, lutein) intake was estimated using the Food Frequency Questionnaire. Sociodemographic and potential covariates were evaluated through questionnaires, anthropometric measurements and blood tests. The association between dietary intakes of antioxidant vitamins and tHcy concentration were evaluated by multiple linear regression analyses after napierian logarithm transformed. Multiple logistic regression models were further used to determine ORs and $95 \% \mathrm{Cls}$. Results The $\beta$ (95\% Cls) of VC intake and tHcy was $-0.050(-0.084$ to -0.016$)$. Compared with the lowest quartile in the fully adjusted model, the ORs (95\% Cls) for HHcy levels across quartiles of dietary VC intake were 0.82 (0.57 to 1.16), 0.49 (0.33 to 0.74 ) and 0.40 (0.22 to 0.74$)$ ( $p$ for trend $=0.001)$. The $\beta$ (95\% Cls) of retinol intake and tHcy was $-0.021(-0.041$ to -0.002$)$ and the ORs $(95 \%$ $\mathrm{Cls})$ in the third quartile of retinol intake was $0.61(0.42$ to 0.86 ), while the effect for the highest quartile was not significant ( $p$ for trend=0.951). No significant association was observed between dietary VE, carotenes and lutein intake and HHcy.

Conclusions A linear inverse association between dietary VC intake and HHcy prevalence, and an L-shaped association between dietary retinol intake and $\mathrm{HHcy}$ prevalence were found in Chinese middle-aged and older adults with hypertension.

\section{INTRODUCTION}

Increasing evidence has shown that elevated total homocysteine (tHcy) levels
Strengths and limitations of this study

- This study focused on the risk of hyperhomocysteinaemia (HHcy) among middle-aged and older adults with hypertension.

- A linear inverse association between dietary vitamin $\mathrm{C}$ intake and HHcy prevalence and the threshold effect of retinol on HHcy was reported in this study.

- Based on a cross-sectional study design, we could only draw a conclusion about correlation, not causation.

- Although some confounding factors were included in the analysis, other potential confounders may exist.

are associated with atherosclerotic cardiovascular diseases (CVD), stroke, peripheral arterial occlusive disease and venous thrombosis. $^{1}{ }^{2}$ Hyperhomocysteinaemia (HHcy) (tHcy $\geq 15 \mu \mathrm{mol} / \mathrm{L}),{ }^{3}$ the result of a disturbed methionine metabolism, may lead to an enhancement of the adverse effects of risk factors like hypertension on human health. ${ }^{4}$ Hypertension is a major risk factor for cardiovascular and chronic kidney disease and a major cause of the global burden of disease and mortality. ${ }^{56}$ It was estimated that the prevalence of hypertension in China in 2015 was 23.2\%, and about 245 million Chinese adults had hypertension. ${ }^{7}$ Notably, the incidence of hypertension with HHcy, or 'H-type hypertension', is significantly higher compared with other countries, representing $75 \%$ of Chinese patients with hypertension. ${ }^{8}$ Thus, the nutritional treatment focus on the attempt to reduce cardiovascular risk by reducing tHcy is important, particularly among patients with hypertension. ${ }^{9}$

Increasing age, male sex, genetic factors, consumption of alcohol or tobacco, kidney function and physical activity are some of the factors associated with tHcy levels. ${ }^{10}$ 
Epidemiological studies and clinical trials have indicated that folate, vitamin $\mathrm{B}_{12}$ and vitamin $\mathrm{B}_{6}$ status, well-known predictors of tHcy, are important for tHcy metabolism. The latest meta-analysis demonstrated that a lower risk of stroke and overall CVD with folic acid supplementation, which may partly contribute to the decrease of tHcy levels. $^{11}$

Folic acid and vitamin $\mathrm{B}_{12}$, which are involved in remethylation of homocysteine to methionine by methionine synthase (MS) and vitamin $B_{6}$, which acts as a cofactor in the transsulfuration of homocysteine to cysteine by cystathionine $\beta$-synthase (CBS) ${ }^{12}$ Folate, a key factor of tHcy metabolism, is very sensitive to free radicals. ${ }^{13-15}$ In addition, MS and CBS were strongly influenced by oxidative stress, which may be associated with dietary antioxidant vitamins and Hcy levels. ${ }^{16}{ }^{17}$ We hypothesised that antioxidant nutrients may regulate tHcy metabolism by influencing methionine-homocysteine cycle. Plasma levels or dietary intake of vitamin $\mathrm{C}(\mathrm{VC})$, vitamin $\mathrm{E}(\mathrm{VE})$ or $\beta$-carotene was inversely associated with tHcy levels, however, the findings were not consistent. ${ }^{18-20}$ Of note, the association has never been investigated among the hypertensive population.

Therefore, this large population-based study aimed to determine the association between the dietary intake of $\mathrm{VC}, \mathrm{VE}$, carotenes, retinol and lutein and the prevalence of HHcy in middle-aged and older men and women with hypertension.

\section{METHODS}

\section{Study design and population}

This study consecutively recruited individuals with hypertension from 60 community health service centres (CHSCs) in the Nanshan district of Shenzhen from July to September of 2013 using a three-stage random sampling method. In the first stage, eight subdistricts were selected in the Nanshan district; in the second stage, 6-8 communities were selected from each su-district using a simple random selection procedure; and in the third stage, individuals with hypertension were selected from each community using isometric random sampling. All subjects were of Chinese ethnicity and had lived in the Nanshan district of Shenzhen for over 6 months. The individuals were invited to visit the CHSCs, where the researcheradministered questionnaire (including the validated Food Frequency Questionnaire (FFQ) ) was conducted, the anthropometric measurements were recorded, and fasting blood samples were collected.

We collected the data of 1802 participants, and excluded 51 participants whose age was not reported or who were aged $\leq 40$ years and 27 underweight participants (body mass index (BMI) $<18.5 \mathrm{~kg} / \mathrm{m}^{2}$ ), as well as participants for whom fasting blood samples $(\mathrm{n}=74)$ and complete dietary surveys $(n=133)$ were not available. We also excluded participants with an extreme dietary energy intake (male: $<800 \mathrm{kcal}$ or $>4000 \mathrm{kcal}$, female: $<700 \mathrm{kcal}$ or $>3500 \mathrm{kcal})(\mathrm{n}=52)$. Finally, 1465 participants were included in the analysis (online supplemental figure 1).

\section{Patient and public involvement}

The development of standardised form is in response to the public health need of preventing stroke among hypertension population. Patients and the public were not involved in the design of the study. The results of our study will be disseminated through open access publications.

\section{Dietary assessment}

The researcher-administered FFQ consisted of 92 food items, which were assembled into 7 food groups: cereals, vegetables, fruits, beans, animal foods, nuts and beverages. The FFQ was based on the national FFQ used in the 2010-2012 China National Nutrition and Health Survey according to the Chinese Nutrition and Health Surveillance in 2010-2012. ${ }^{21}$ Participants were asked to recall the consumption of each item during the past year, including the type of food, frequency and amount. Food weight maps were available for participants to estimate their portion size. Primary data obtained from FFQs were doubly entered into the EpiData V.3.0 software by two trained technicians to verify accuracy. Dietary energy and other nutrients were calculated based on the Chinese Food Composition Database. ${ }^{22}{ }^{23}$ Dietary intake of VC, $\mathrm{VE}$, carotenes (consist of $\alpha$-carotene, $\beta$-carotene and $\beta$-cryptoxanthin), and retinol were calculated based on the Chinese Food Composition Table 2002. ${ }^{22}$ We report carotenes and retinol separately, because we could not get the accurate calculation for vitamin $\mathrm{A}$, as the data for carotenes were combined with different carotene, rather than separate one. Dietary intake of lutein was calculated based on the food composition table of vegetables, fruits, eggs and nuts that contain large amounts of lutein, according to Chinese Dietary Reference Intakes 2013. ${ }^{24}$ The intake of all dietary nutrients and carotenoids was adjusted for energy using the residual method. ${ }^{25}$ The second FFQ was conducted 3 weeks after the completion of the first FFQ among 108 participants. The intraclass correlation coefficients of two administrations of FFQ for nutrients of VC, VE, carotenes, retinol, lutein were 0.395 , $0.477,0.355,0.551$ and 0.350 , and were all statistically significant.

\section{Assessment of other covariates}

Participants' sociodemographic characteristics (eg, age and sex), lifestyle factors (eg, sedentary time), history of chronic diseases and medication and supplement use status were collected. Sedentary time consisted of time spent watching TV and sitting, which were combined into one variable with two categories, $<3$ hours/day and $\geq 3$ hours/day, based on a median sedentary time of 3 hours/day. The history of cardiovascular events including coronary heart disease, cerebral haemorrhage, cerebral thrombosis and the history of diabetes and kidney disease were recorded for each participant. 
The prescription use status was classified into two groups (yes or no) corresponding to whether the participant was taking any type or quantity of drugs, including antihypertensive drugs, antihyperglycaemic drugs, lipid-lowering drugs or tHcy-lowering drugs. Supplement use consisted of vitamin A, retinol, B vitamins, VC, VE and folic acid was included.

\section{Anthropometric measurements}

Height and weight and waist circumference (WC) were measured by specialists. BMI was calculated as weight $(\mathrm{kg}) /$ height $\left(\mathrm{m}^{2}\right)$. Systolic blood pressure $(\mathrm{SBP})$ and diastolic blood pressure were measured from the right arm of participants in a seated position after a sufficient rest period using a mercury sphygmomanometer in the morning. Blood pressure was measured manually and recorded as the average of three measurements.

\section{Laboratory tests and outcomes}

Morning fasting elbow vein blood samples were required to fast overnight (at least 10 hours) were collected from the participants at the CHSCs and transported under refrigerated conditions to a clinical laboratory of the Nanshan Centre for Chronic Disease Control within 2 hours. The blood specimens were collected in a $5 \mathrm{~mL}$ EDTA vacuum tube. Blood samples were collected through deposition and centrifugation for ten minutes at $3000 \mathrm{r} / \mathrm{min}$ at room temperature. The concentrations of plasma tHcy, fasting plasma glucose (FPG), total cholesterol, triglyceride (TG), low-density lipoprotein cholesterol, uric acid and creatinine were assessed on the day of blood collection using enzymatic methods via an auto-analyser (HITACHI 7080). The interday quality control assessments met the standard during the analysis. HHcy was defined as plasma tHcy concentration $\geq 15 \mu \mathrm{mol} / \mathrm{L}$.

\section{Statistical analysis}

Demographic characteristics were described by means \pm SDs for normally distributed data, medians (IQRs) for non-normally distributed data and numbers (percentages) for categorical data. The differences between males and females were compared using the t-test for normally distributed variables, the Kruskal-Wallis rank test for nonnormally distributed variables, and the $\chi^{2}$ test for categorical variables.

Both dietary intakes of antioxidant vitamins and tHcy concentration were napierian logarithm (ln)-transformed to improve normality before analyses and categorised into quartiles. The associations between dietary antioxidant vitamins intake and the prevalence of HHcy were analysed using multiple logistic regression models, with the lowest quartile as the reference category. Confounding variables were selected based on the minimal sufficient adjustment recommended by the Directed Acyclic Graph, created in the online software Dagitty V.3.0 (online supplemental figure 2). The selected potential confounders included age, sex ('male' as the reference), BMI, sedentary time ('<3hours/day' as the reference), the intakes of folate, vitamin $\mathrm{B}_{6}$ and vitamin $\mathrm{B}_{12}$, supplement use ('no' as the reference) and the history of cardiovascular events ('no' as the reference). Linear trends were tested by creating a continuous variable for dietary antioxidant vitamins intake using the median value for each quartile. The sensitive analyses between dietary antioxidant vitamins intake and HHcy prevalence were applied among the population who had never suffered cardiovascular events, or among the population who never use the tHcy-lowering drug.

To further explore the nonlinearity of the relationship between dietary antioxidant vitamins intake and high tHcy, we used restricted cubic spline with four knots at the 5th, 35th, 65th and 95th percentiles of dietary antioxidant vitamins intake.

To evaluate the modification effect by some potential prevalence factors of HHcy, including sex (male or female) and BMI $\left(<24 \mathrm{or} \geq 24 \mathrm{~kg} / \mathrm{m}^{2}\right)$, stratified analyses were conducted by these potential factors and estimated $p$ values for interaction terms.

All data were analysed using the R Foundation V.3.1.2 and Empower $\mathrm{X} \& \mathrm{Y}$ Solutions. Statistical significance was considered when $\mathrm{p}<0.05$ (two sided).

\section{RESULTS}

In this cross-sectional study, 1465 participants (male: 729, female: 736) were included in the analysis, and the mean \pm SD of age and BMI was $62.0 \pm 10.7$ years, and $24.9 \pm 3.5 \mathrm{~kg} / \mathrm{m}^{2}$. The tHcy levels of the participants was $14.63 \pm 9.06 \mu \mathrm{mol} / \mathrm{L}$, and the number (percentage) of HHcy was 469 (32.0\%). The participants with HHcy were older and more male, and have higher WC, SBP, tHcy, uric acid, creatinine and higher percentages of history of cardiovascular events (including coronary heart disease, cerebral haemorrhage, cerebral thrombosis) and kidney disease, and have lower FPG level (all p<0.05) (table 1).

The dietary intakes of nutrients were shown in table 2 . The median (IQR) of dietary antioxidant vitamins intake were as follows: VC $153.5(91.2,240.9) \mathrm{mg} /$ day, VE 25.0 $(19.3,31.9) \mathrm{mg} /$ day, carotenes $3.3(1.8,5.6) \mathrm{mg} /$ day, retinol $138.3(78.0,283.6) \mu \mathrm{g} /$ day and lutein 9.5 (5.2, 14.8) $\mathrm{mg} /$ day. Among the participants with HHcy, the intake of carbohydrate was higher, and the intake of fruit, protein, fibre, retinol, folate, vitamin $\mathrm{B}_{12}$, saturated fatty acid, selenium, magnesium, zinc was lower, and the percentage of supplement use was lower (all $\mathrm{p}<0.05$ ).

The association between dietary antioxidant vitamins intake and tHcy levels are shown in table 3. The inverse association between VC intake and tHcy concentration after ln-transformed in the fully adjusted model, and the $\beta(95 \%$ CIs $)$ was $-0.050(-0.084$ to -0.016$)$, which was consistent with the logistic regression as categorical variables. In model II, the significant association was found in the third and highest quartile of VC intake, and the ORs (95\% CIs) were 0.49 (0.33 to 0.74$)$ and $0.40(0.22$ to 0.74$)\left(\mathrm{p}_{\text {for trend }}=0.001\right)$. The retinol intake was also inversely associated with tHcy, as the $\beta$ (95\% CIs) was 
Table 1 Basic characteristics of the participants*

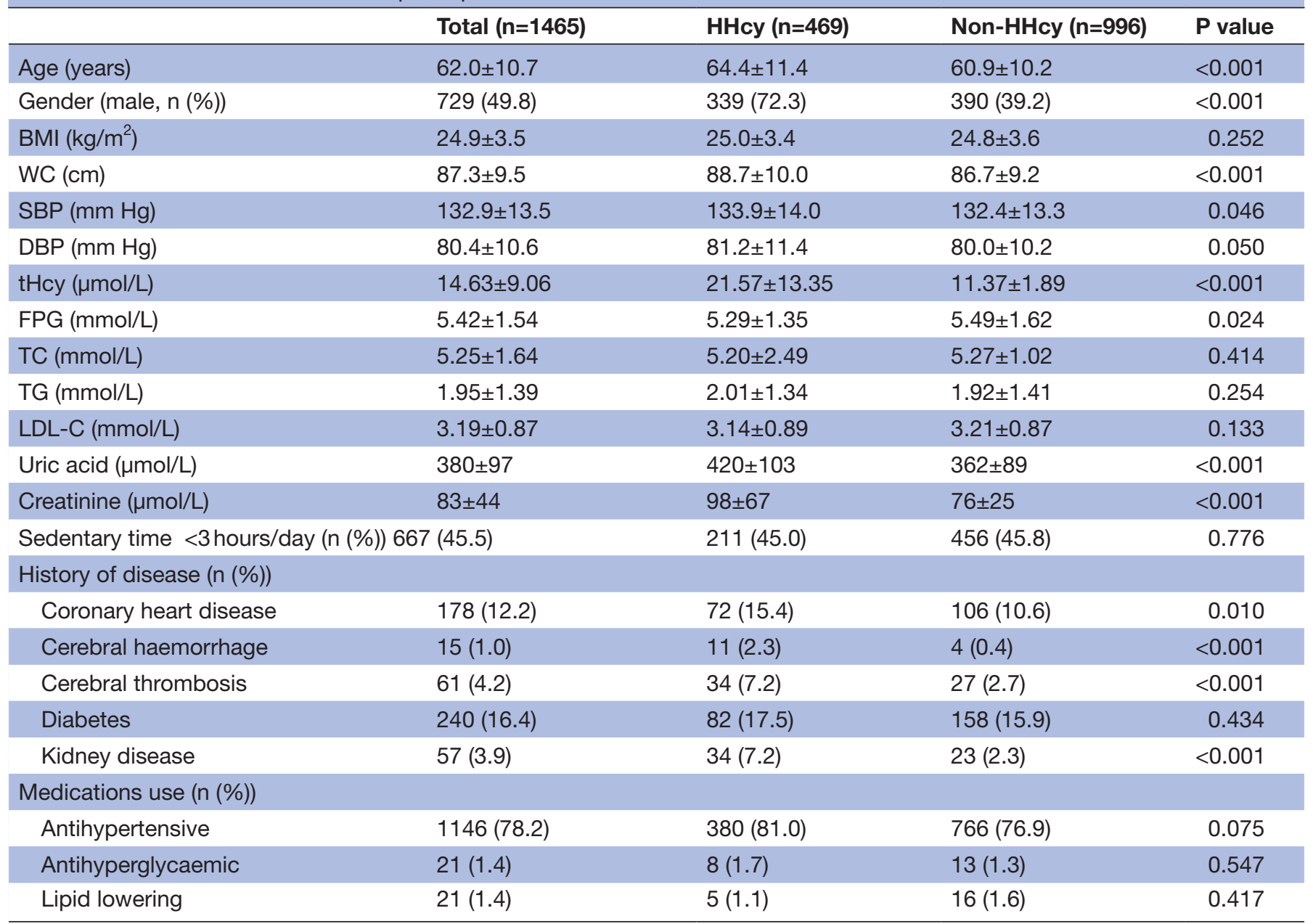

*Values are mean \pm SD or median (IQR) or number (percentage).

BMI, body mass index; DBP, diastolic blood pressure; FPG, fasting plasma glucose; HHcy, hyperhomocysteinaemia; LDL-C, lowdensity lipoprotein cholesterol; SBP, systolic blood pressure; TC, total cholesterol; TG, triglyceride; tHcy, total homocysteine; WC, waist circumference.

$-0.021(-0.041$ to -0.002$)$. After adjusting for age, sex and BMI, the ORs (95\% CIs) for HHcy prevalence across quartiles of retinol intake were $1.00,0.84(0.61$ to 1.16$)$, $0.50(0.35$ to 0.70$)$ and $0.55(0.40$ to 0.77$)\left(\mathrm{P}_{\text {for trend }}=\right.$ $0.003)$. However, in model II, the significant association was only found in the third quartile of retinol intake, and the ORs $(95 \%$ CIs ) was 0.61 (0.42 to 0.86$)\left(\mathrm{P}_{\text {for trend }}\right.$ $=0.951)$. The non-significant association between dietary intake of VE, carotenes and lutein and HHcy prevalence was found. The results were similar in the sensitivity analyses that excluded participants with cardiovascular events or who use the tHcy-lowering drug (see online supplemental table 1).

After fully adjusting for the potential confounders, the association between dietary antioxidant vitamins intake and the prevalence of HHcy were shown in figure 1. From the cubic splines, we noted that the linear inverse trend of $\mathrm{VC}$ intake and HHcy prevalence ( $p$ for overall association $=0.016$, $p$ for nonlinearity $=0.055$ ) and the L-shaped relationship of retinol intake and HHcy prevalence ( $p$ for overall association $=0.011, \mathrm{p}$ for nonlinearity $=0.020$ ), which were consistent with the results of logistic regression analyses. The threshold analysis showed that the ORs (95\% CIs) of HHcy was 0.995 (0.991, 0.995) ( $\mathrm{p}=0.005)$ when retinol intake was lower than $147.2 \mu \mathrm{g} /$ day and $1.000(1.000,1.001)(p=0.094)$ when retinol intake was more than $147.2 \mu \mathrm{g} /$ day and the $\mathrm{p}$ value of log-likelihood ratio was 0.003 . The non-association between carotenes, lutein and VE and HHcy was not shown.

In stratified analyses, the association between dietary $\mathrm{VC}$ and retinol intake and $\mathrm{HHcy}$ prevalence were not significantly modified by sex (male or female), BMI $\left(<24\right.$ or $\left.\geq 24 \mathrm{~kg} / \mathrm{m}^{2}\right) \quad\left(\right.$ all $\mathrm{p}_{\text {for interaction }}$ were $\left.>0.05\right)$ (figure 2$)$. Similar results of stratified analyses of carotenes, lutein and VE were not shown.

\section{DISCUSSION}

In this community-based cross-sectional study, we observed some of the antioxidant vitamins intakes were significantly correlated with the prevalence of HHcy. After adjusting potential confounders, a linear inverse 
Table 2 The dietary intake of food and nutrients of the participants*

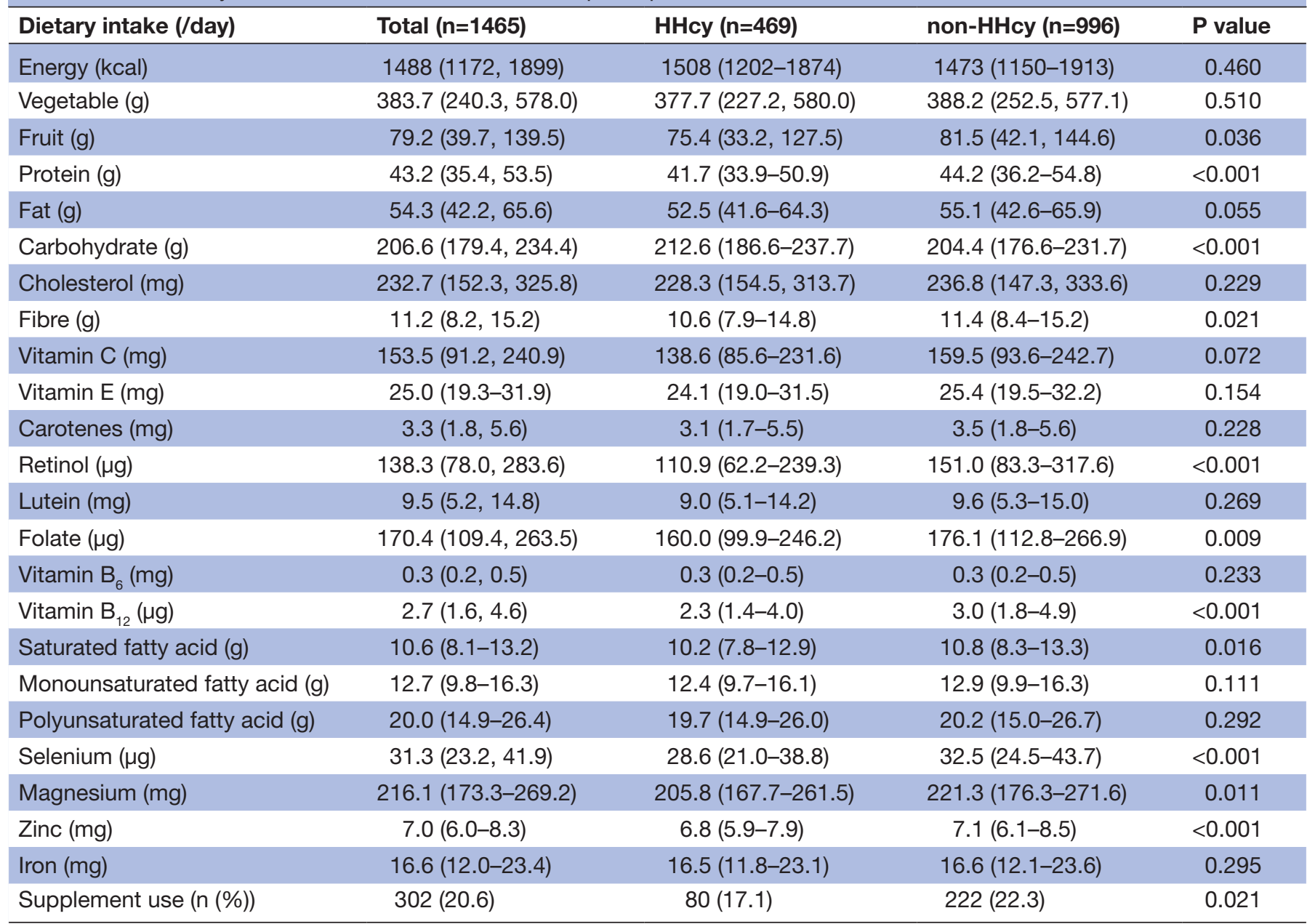

*Values are median (IQR) or number (percentage).

HHcy, hyperhomocysteinaemia.

association between VC intake and HHcy prevalence, and an L-shaped relationship between retinol intake and HHcy prevalence were found, which were not modified by sex or BMI. However, the non-significant effect of VE, carotenes and lutein on HHcy was detected.

Numerous studies have suggested that HHcy may be a modifiable risk factor for CVD, especially for stroke. In the past decades, there were many clinical trials aimed to show the effect of folate and vitamins $B_{12}$ and $B_{6}$ supplement on lowering the levels of tHcy, however, the negative results were found in most studies. ${ }^{26}$ The potential reason may be correlated with the harmful effect of unmetabolised excessive folate or the cyanide and thiocyanate from excessive cyanocobalamin. In China, where folate fortification has not been implemented, folic acid significantly reduced the risk of stroke was observed from the China Stroke Primary Prevention Trial (CSPPT). ${ }^{27}$ Thus, appropriate B vitamins therapy is of great importance for lowering tHcy levels in stroke prevention.

In the past decades, several researches reported the potential association between plasma levels or intake of different antioxidant vitamins and tHcy levels. ${ }^{18} 19$ 28-30
In 1999, Brude IR et al observed the inverse association between plasma tHcy concentration and dietary intake of vegetables, $\mathrm{VC}$ and $\beta$-carotene from 41 participants. ${ }^{28}$ In addition, dietary intake of retinol equivalents, $\beta$-carotene and VC were inversely correlated with plasma tHcy levels, after adjustment for dietary B-vitamins, but not after additional adjustment for plasma folate and vitamin $\mathrm{B}_{12}{ }^{19}$ What's more, the study focused on the effect of antioxidant vitamins on the plasma tHcy levels in a free-living elderly population found that plasma VC, rather than the intake and supplementation of $\mathrm{VC}$, showed a negative association with tHcy in simple regression analysis, and also found that the plasma levels, as well as the intake and supplementation of vitamin $\mathrm{E}$, and $\beta$-carotene were not associated with tHcy. ${ }^{18}$ Similarly, the cross-sectional National Health And Nutrition Examination Survey 1999-2002 study found that dietary VC and VE intake were associated with a lower prevalence of elevated blood tHcy concentration, whereas no association between dietary carotenes intake and tHcy was detected. ${ }^{29}$ In addition, Ullegaddi et al found that significant reductions in plasma homocysteine in the group with antioxidant 
Table $3 \quad \beta(95 \% \mathrm{Cls})$ and ORs (95\% Cls) of dietary intakes of antioxidant vitamins and homocysteine levels among the middle-aged and older hypertensive participants

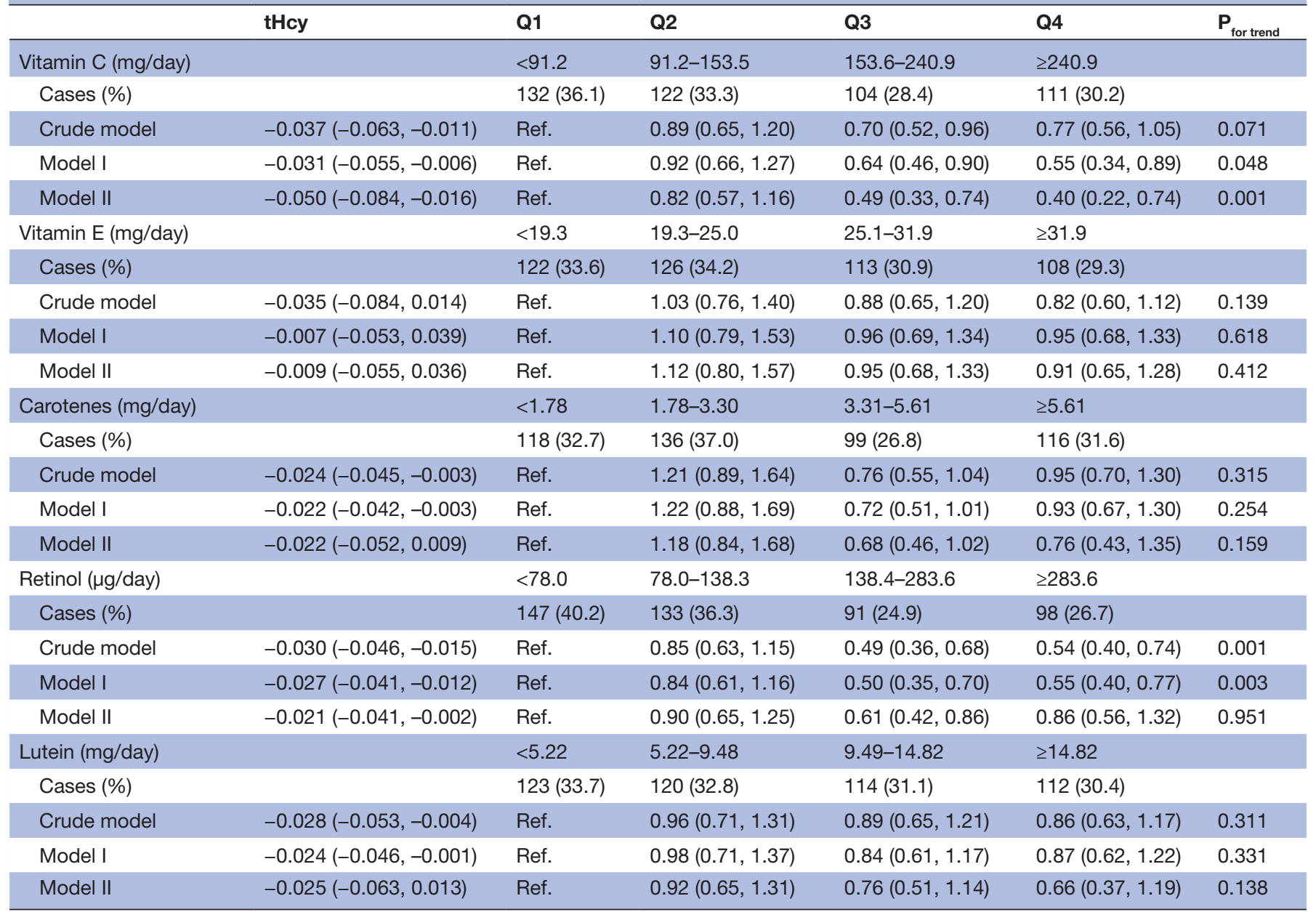

Model I: Adjusted for age, sex ('male' as the reference) BMI.

Model II: Adjusted for variables in model I and further adjusted for sedentary time ('<3 hours/day' as the reference), the intakes of folate, vitamin $B_{6}$ and vitamin $\mathrm{B}_{12}$, supplement use ('no' as the reference) and the history of cardiovascular events ('no' as the reference).

$\mathrm{BMI}$, body mass index; tHcy, total homocysteine.

treatment (vitamins $\mathrm{C}$ and $\mathrm{E}$ ) combination with B-vitamin, compared with the group with B-vitamin alone, ${ }^{30}$ which support the importance of antioxidant vitamins on tHcy metabolism.

Consistent with our findings, these studies have a common conclusion that VC intake was inversely correlated with tHcy levels. Given the report of an interaction of $\mathrm{VC}$ and folate, ${ }^{31}$ Magana et al found the underlying molecular mechanisms that VC activates the folate-mediated one-carbon cycle in C2C12 myoblasts. ${ }^{32}$ Thus, VC has been explored as an attractive factor to increase circulating levels of folic acid and to reduce Hcy levels.

We found an L-shaped association between dietary retinol intake and high tHcy prevalence, which meant that if the retinol intake was low, the risk of HHcy was decreased as retinol intake increased, but the risk was not changed when retinol intake reached certain level, which was more than $147.2 \mu \mathrm{g} /$ day among the participants in this study. The dietary intake of retinol equivalents was inversely correlated with plasma tHcy levels after adjustment for dietary B-vitamins. ${ }^{19}$ Retinol, a preformed vitamin A, plays an important role in vision, cellular differentiation and proliferation, as well as the immune system regulation. In addition, there is increasing evidence indicates that retinol seems to inhibit thrombosis ${ }^{33}$ and inflammation effects, ${ }^{34}$ which indicates retinol is emerging as a factor of interest to CVD. Brazionis et al reported that plasma retinol was a novel marker for CVD mortality in Australian adults, with an inverse association between plasma retinol in the middle tertile and 5-year CVD mortality. ${ }^{35}$ Similarly, a strong association between low retinol and the risk of sudden cardiac death was examined. ${ }^{36}$ In addition, a nested case-control study showed a significant inverse association between plasma retinol and the risk of first stroke among Chinese hypertensive adults from the CSPPT,${ }^{37}$ which may due to relatively low baseline retinol concentrations (median: $67.5 \mu \mathrm{g} / \mathrm{dL}$ ). Besides, the interaction of retinol and tHcy on CVD risk was also reported. $\mathrm{Yu} e t a \hat{l}^{87}$ showed the inverse effect between plasma retinol 

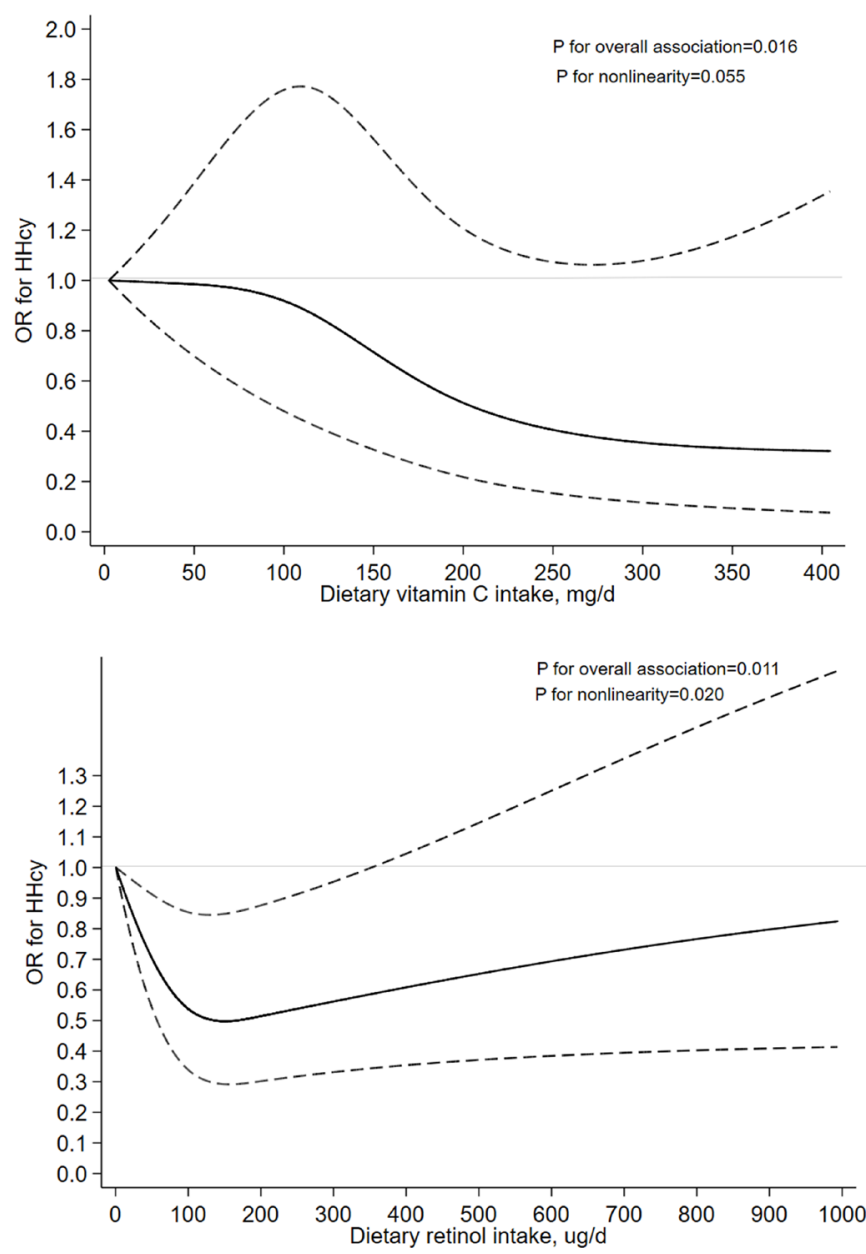

Figure 1 Restricted cubic spline analyses illustrating the shapes of multivariable association between dietary vitamin $\mathrm{C}$ intake (A), or dietary retinol intake (B) and HHcy. Adjusted for age, sex ('male' as the reference), BMI, sedentary time ('<3 hours/day' as the reference), supplement use ('no' as the reference), dietary intake of folate, vitamin $B_{6}$ and vitamin $B_{12}$, the history of cardiovascular events ('no' as the reference). BMI, body mass index; HHcy, hyperhomocysteinaemia.

and first stroke was stronger among the participants whose tHcy $<10 \mu \mathrm{mol} / \mathrm{L}$ than whose tHcy $\geq 10 \mu \mathrm{mol} / \mathrm{L}$. Olsen $e t$ $a l^{38}$ found that the plasma tHcy was associated with acute myocardial infarction only in the upper Vit-A tertile, and the potential mechanisms may include inflammation and lipid metabolism, which may be partly interpreted with the high intake of retinol ( $1576 \mu \mathrm{g} \mathrm{RAE} /$ day).

In addition, the dietary source of retinol may contribute to the non-significant effect of retinol in the highest quartile in the fully adjusted model, as it is present in animal-based foods, particularly in liver and whole milk. In this study, we found the TG level was gradually increased with the increase of retinol (data not shown). High retinol intake may alter lipid metabolism by increasing TG level, which may impact the tHcy metabolism. ${ }^{39}$

At present, a few studies demonstrate the complicated relationship between retinol, tHcy and CVD risk, but the underlying mechanism has not yet been clarified. ${ }^{40}$ The
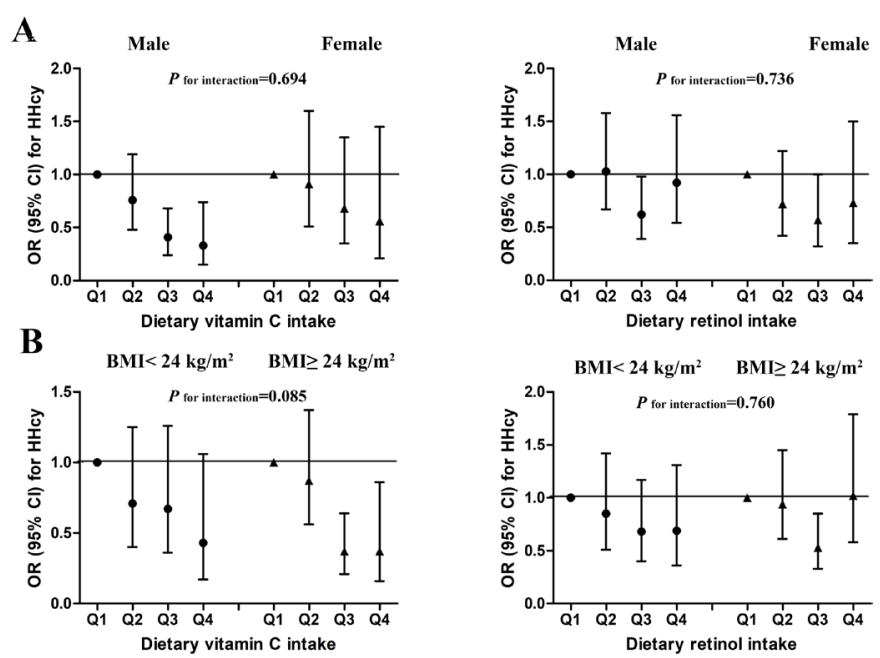

Figure 2 ORs and 95\% Cls for hyperhomocysteinaemia (HHcy) prevalence according to vitamin $\mathrm{C}$, retinol intake in subgroups, stratified by sex (A), BMI (B). Adjusted for age, sex (except sex subgroup), BMI (except BMI subgroup), sedentary time ('<3 hours/day' as the reference), supplement use ('no' as the reference), dietary intake of folate, vitamin $B_{6}$ and vitamin $B_{12}$, the history of cardiovascular events ('no' as the reference). BMI, body mass index.

antioxidant activity of retinol may be a plausible mechanism that links the effect of retinol on tHcy levels, as retinol is essential for the maintenance of immune function and antioxidant defence. ${ }^{41} 42$

However, we found no relationship between HHcy prevalence and dietary intake of VE. The aforementioned study has found significant inverse associations between plasma tHcy and dietary intake of VE, but the effect was not significant after adjusting B-vitamins intake, which was consistent with our finding. ${ }^{19}$ On the contrary, dietary VE ( $\alpha$-tocopherol) intake was associated with a lower risk of elevated blood tHcy concentration among US adults. ${ }^{29}$

Epidemiological studies have reported the positive role of carotenoids on human health. In this study, we focused on the effect of carotenes ( $\beta$-carotene, $\alpha$-carotene and $\beta$-cryptoxanthin) and lutein on tHcy, however, the negative results were found. Many of the aforementioned studies reported the protective effect of $\beta$-carotene by lowering tHcy concentration, ${ }^{19} 28$ but the risk of tHcy $>13 \mu \mathrm{mol} / \mathrm{L}$ was associated with the total carotene intake from diet plus supplement use, rather than the only intake from diet. ${ }^{29}$ The negative finding of lutein cannot be compared because of a lack of previously reported data. Thus, more prospective cohort studies and randomised double trials are warranted to indicate the relationship of antioxidant vitamins with HHcy risk.

The strength of this study is the face-to-face researcheradministered FFQ survey. First, to our knowledge, this is the first study to demonstrate an association of dietary intake of antioxidant vitamins with HHcy prevalence that was conducted in both a male and female hypertensive 
population. Then, the researcher-administered face-toface interview was considered by validated FFQ, which was designed to evaluate dietary intake and gave full consideration to eating habits and food nutrient composition in the Chinese population.

Our study has several limitations. First, based on a crosssectional study design, we cannot draw a conclusion about causality. The strong effects observed in this study may be enhanced because of selection effects, because hypertensive individuals may have made changes to their diet in response to diagnosis. Second, we adjusted the dietary intake of folate, $\mathrm{B}_{12}, \mathrm{~B}_{6}$, rather than plasma concentrations, which may could not eliminate confounding effect. Just like Konstantinova $\mathrm{SV}^{19}$ reported, the inverse correlation of dietary retinol intake and plasma tHcy disappeared after adjustment for plasma folate and vitamin $B_{12}$. Then, we investigated the participants whether they took dietary supplements, while the detailed doses were not recorded. Therefore, we are not able to eliminate the possible association between supplement use and HHcy prevalence. Nevertheless, the study reported that there was some potential benefit from the antioxidant supplementation on plasma tHcy concentration, ${ }^{29}$ which could stabilise the positive results in this study. In addition, although some confounding factors were included in the analysis, other potential confounders may exist. For instance, the influence of smoking and drinking on HHcy was not assessed because of the lack of information about the status of smoking and drinking.

In conclusion, we found dietary intake of $\mathrm{VC}$ and retinol was inversely associated with HHcy prevalence in middleaged and older adults with hypertension after adjusting the potential confounders. Our findings have provided suggestive evidence of an inverse relationship between certain antioxidant intake and HHcy, which should be the impetus for longitudinal and random control trails to verify the relationship and direction and to elucidate the underlying mechanisms in the future.

\section{Author affiliations \\ ${ }^{1}$ Department of Tumor, Injury and Nutrition, Nanshan Centre for Chronic Disease Control, Shenzhen, Guangdong, People's Republic of China \\ ${ }^{2}$ Department of Nutrition and Food Hygiene, School of Public Health, Tongji Medical College, Huazhong University of Science and Technology, Wuhan, Hubei, People's Republic of China \\ ${ }^{3}$ Department of Public Health, Jining Medical College, Jining, Shandong, People's Republic of China}

Acknowledgements The authors are grateful to the individuals who participate in this study. We also thank the investigators, staff and participants from the community health service centres for their assistance.

Contributors The authors' responsibilities were as follows: LH conceived and designed the study and critically revised the manuscript. XP and QG analysed the data and wrote the paper. JZ participated in the laboratory assay. JM and DZ collected the data and revised the manuscript. All authors read and approved the final version of the manuscript.

Funding The present study was jointly supported by the National Natural Science Foundation of China (No. 81573149), the Health and Family Commission of Shenzhen Municipality Funded Project (No. 201502017 and SZSJ2017001) and Nanshan District, Shenzhen Science and Technology Innovation Bureau funded projects (No. 2015064).
Competing interests None declared.

Patient consent for publication Not applicable.

Ethics approval The survey protocol was approved by the Ethics Committee of the Shenzhen Nanshan Centre for Chronic Disease Control (ID: II20190003), and all participants provided written informed consent before enrolment.

Provenance and peer review Not commissioned; externally peer reviewed.

Data availability statement No data are available.

Supplemental material This content has been supplied by the author(s). It has not been vetted by BMJ Publishing Group Limited (BMJ) and may not have been peer-reviewed. Any opinions or recommendations discussed are solely those of the author(s) and are not endorsed by BMJ. BMJ disclaims all liability and responsibility arising from any reliance placed on the content. Where the content includes any translated material, BMJ does not warrant the accuracy and reliability of the translations (including but not limited to local regulations, clinical guidelines, terminology, drug names and drug dosages), and is not responsible for any error and/or omissions arising from translation and adaptation or otherwise.

Open access This is an open access article distributed in accordance with the Creative Commons Attribution Non Commercial (CC BY-NC 4.0) license, which permits others to distribute, remix, adapt, build upon this work non-commercially, and license their derivative works on different terms, provided the original work is properly cited, appropriate credit is given, any changes made indicated, and the use is non-commercial. See: http://creativecommons.org/licenses/by-nc/4.0/.

ORCID iD

Liping Hao http://orcid.org/0000-0001-8844-0892

\section{REFERENCES}

1 Price BR, Wilcock DM, Weekman EM. Hyperhomocysteinemia as a risk factor for vascular contributions to cognitive impairment and dementia. Front Aging Neurosci 2018;10:350.

2 Saposnik G, Ray JG, Sheridan P, et al. Homocysteine-Lowering therapy and stroke risk, severity, and disability: additional findings from the hope 2 trial. Stroke 2009;40:1365-72.

3 Guo H, Chi J, Xing Y, et al. Influence of folic acid on plasma homocysteine levels \& arterial endothelial function in patients with unstable angina. Indian J Med Res 2009;129:279-84.

4 Baszczuk A, Kopczyński Z. [Hyperhomocysteinemia in patients with cardiovascular disease]. Postepy Hig Med Dosw 2014;68:579-89.

5 Forouzanfar MH, Liu P, Roth GA, et al. Global burden of hypertension and systolic blood pressure of at least 110 to $115 \mathrm{MM} \mathrm{Hg}, 1990-$ 2015. JAMA 2017;317:165-82.

6 Carey RM, Muntner P, Bosworth HB, et al. Reprint of: Prevention and Control of Hypertension: JACC Health Promotion Series. J Am Coll Cardiol 2018;72:2996-3011.

7 Wang Z, Chen Z, Zhang L, et al. Status of hypertension in China: results from the China hypertension survey, 2012-2015. Circulation 2018;137:2344-56.

8 Liu LS. [Chinese guidelines for the management of hypertension]. Zhonghua Xin Xue Guan Bing Za Zhi 2010;2011:579-615.

9 Azzini E, Ruggeri S, Polito A. Homocysteine: its possible emerging role in at-risk population groups. Int J Mol Sci 2020;21:1421.

10 Shenoy V, Mehendale V, Prabhu K, et al. Correlation of serum homocysteine levels with the severity of coronary artery disease. Indian J Clin Biochem 2014;29:339-44.

$11 \mathrm{Li}$ Y, Huang T, Zheng Y, et al. Folic acid supplementation and the risk of cardiovascular diseases: a meta-analysis of randomized controlled trials. J Am Heart Assoc 2016;5:e003768.

12 Joseph J, Loscalzo J. Methoxistasis: integrating the roles of homocysteine and folic acid in cardiovascular pathobiology. Nutrients 2013;5:3235-56.

13 Odin E, Carlsson G, Frösing R, et al. Chemical stability and human plasma pharmacokinetics of reduced folates. Cancer Invest 1998;16:447-55.

14 Lucock MD, Priestnall M, Daskalakis I, et al. Nonenzymatic degradation and salvage of dietary folate: physicochemical factors likely to influence bioavailability. Biochem Mol Med 1995;55:43-53.

15 Verlinde PHCJ, Oey I, Hendrickx ME, et al. L-ascorbic acid improves the serum folate response to an oral dose of [6S]5 -methyltetrahydrofolic acid in healthy men. Eur J Clin Nutr 2008;62:1224-30.

16 Zou C-G, Banerjee R. Homocysteine and redox signaling. Antioxid Redox Signal 2005;7:547-59. 
17 Waly M, Power-Charnitsky V-A, Hodgson N, et al. Alternatively spliced methionine synthase in SH-SY5Y neuroblastoma cells: cobalamin and GSH dependence and inhibitory effects of neurotoxic metals and thimerosal. Oxid Med Cell Longev 2016;2016:1-11.

18 Breilmann J, Pons-Kühnemann J, Brunner C, et al. Effect of antioxidant vitamins on the plasma homocysteine level in a free-living elderly population. Ann Nutr Metab 2010;57:177-82.

19 Konstantinova SV, Vollset SE, Berstad P, et al. Dietary predictors of plasma total homocysteine in the Hordaland homocysteine study. $\mathrm{Br}$ J Nutr 2007;98:201-10.

20 Racek J, Rusnáková H, Trefil L, et al. The influence of folate and antioxidants on homocysteine levels and oxidative stress in patients with hyperlipidemia and hyperhomocysteinemia. Physiol Res 2005;54:87-95.

21 Zhao L, Ma G, Piao J, et al. [Scheme of the 2010-2012 Chinese nutrition and health surveillance]. Zhonghua Yu Fang Yi Xue Za Zhi 2016;50:204-7.

22 Yang Y, Wang G, Pang X. China food composition 2002. Beijing: Peking University Medical Center Press, 2002.

23 Yang Y. China food composition 2004. Beijing: Peking University Medical Press, 2004.

24 Chinese Society of Nutrition. Chinese dietary reference intakes 2013. Beijing: Science Press, 2014.

25 Willett W, Stampfer MJ. Total energy intake: implications for epidemiologic analyses. Am J Epidemiol 1986;124:17-27.

26 Spence JD. Homocysteine lowering for stroke prevention: unravelling the complexity of the evidence. Int J Stroke 2016;11:744-7.

27 Huo Y, Li J, Qin X, et al. Efficacy of folic acid therapy in primary prevention of stroke among adults with hypertension in China: the CSPPT randomized clinical trial. JAMA 2015;313:1325-35.

28 Brude IR, Finstad HS, Seljeflot I, et al. Plasma homocysteine concentration related to diet, endothelial function and mononuclear cell gene expression among male hyperlipidaemic smokers. Eur $J$ Clin Invest 1999;29:100-8

29 Floegel A, Chung S-J, von Ruesten A, et al. Antioxidant intake from diet and supplements and elevated serum C-reactive protein and plasma homocysteine concentrations in US adults: a cross-sectional study. Public Health Nutr 2011;14:2055-64.

30 Ullegaddi R, Powers HJ, Gariballa SE. Antioxidant supplementation with or without B-group vitamins after acute ischemic stroke: a randomized controlled trial. JPEN J Parenter Enteral Nutr 2006;30:108-14.

31 Lucock M, Yates Z, Boyd L, et al. Vitamin C-related nutrient-nutrient and nutrient-gene interactions that modify folate status. Eur J Nutr 2013;52:569-82

32 Magana AA, Reed RL, Koluda R, et al. Vitamin C activates the folate-mediated one-carbon cycle in $\mathrm{C} 2 \mathrm{C} 12$ myoblasts. Antioxidants 2020;9:217.

33 Horie S, Kizaki K, Ishii H, et al. Retinoic acid stimulates expression of thrombomodulin, a cell surface anticoagulant glycoprotein, on human endothelial cells. differences between up-regulation of thrombomodulin by retinoic acid and cyclic AMP. Biochem $J$ 1992;281:149-54.

34 Swamidas GP, Basaraba RJ, Baybutt RC. Dietary retinol inhibits inflammatory responses of rats treated with monocrotaline. J Nutr 1999;129:1285-90.

35 Brazionis L, Walker KZ, Itsiopoulos C, et al. Plasma retinol: a novel marker for cardiovascular disease mortality in Australian adults. Nutr Metab Cardiovasc Dis 2012;22:914-20.

36 Espe KM, Raila J, Henze A, et al. Impact of vitamin A on clinical outcomes in haemodialysis patients. Nephrol Dial Transplant 2011;26:4054-61.

$37 \mathrm{Yu} \mathrm{Y}$, Zhang $\mathrm{H}$, Song $\mathrm{Y}$, et al. Plasma retinol and the risk of first stroke in hypertensive adults: a nested case-control study. Am J Clin Nutr 2019;109:449-56.

38 Olsen T, Vinknes KJ, Svingen GF, et al. The risk association of plasma total homocysteine with acute myocardial infarction is modified by serum vitamin A. Eur J Prev Cardiol 2018;25:1612-20.

39 Puddu PE. Serum vitamin A, total homocysteine and acute myocardial infarction: is there a causal association? Eur J Prev Cardiol 2018;25:1607-11.

40 Momin M, Jia J, Fan F, et al. Relationship between plasma homocysteine level and lipid profiles in a community-based Chinese population. Lipids Health Dis 2017:16:54.

41 da Silva R, dos Santos-Valente EC, Burim Scomparini F, et al. The relationship between nutritional status, vitamin $A$ and zinc levels and oxidative stress in patients with ataxia-telangiectasia. Allergol Immunopathol 2014;42:329-35.

42 Ferreira CA, de Souza FIS, Melges APB, et al. Retinol, beta-carotene oxidative stress, and metabolic syndrome components in obese asthmatic children. Pediatr Allergy Immunol 2014;25:292-4. 